\title{
Mental Files and their Identity Conditions
}

\author{
Thea Goodsell \\ University of Oxford \\ BIBLID [0873-626X (2013) 36; pp. 177-190]
}

It is increasingly common for a thinker's capacity for singular thought to be described in terms of that thinker having 'mental files' on the individuals thought about. ${ }^{1}$ A mental file is supposed to have a dual role: to be thinker T's repository of information about an individual $a$, and to be the mode of presentation under which T thinks about $a$.

Mental files seem to offer an appealing account of what modes of presentation are. They allow us to think about modes of presentation by analogy with familiar paper-based or electronic filing systems. And because files are supposed to be psychologically-realised clusters of information, they offer an abstracta-free account of modes of presentation. Moreover, the suggestion that there are files is given 'empirical bite' (Recanati 2012: viii) by talk of 'files' in psychology (e.g. Kahneman et al. 1984, 1992) and linguistics (e.g. Heim 1983, 1988).

Positing mental files appears to require making substantive claims about mental representation. Nonetheless, the suggestion that singular thoughts deploy mental files is usually made in just a paragraph or two. ${ }^{2}$ Recanati's Mental Files provides a welcome contrast. In it, he develops by some way the fullest account of mental files to date. He defends files from certain objections, and works through in detail how files can be used to resolve certain long-standing puzzles in philosophy of language.

According to Recanati's 'indexical' model, files are typed by the epistemically rewarding (ER) relation they are based on (Chapter 5).

${ }^{1}$ E.g. Strawson 1974; Perry 1980; Forbes 1990; Segal 2001; Dickie 2010; Jeshion 2010.

${ }^{2}$ E.g. Forbes 1990, Segal 2001, Jeshion 2010.

Disputatio, Vol. V, No. 36, October 2013 
ER relations range from the simple (e.g. currently visually-attending an object), to the composite (e.g. currently visually-attending an object and remembering having visually-attended it in the past), right up to the higher-order relation that holds if a subject stands in any particular epistemic relation to an object.

A primary role for a file is to collect information about an object sourced through the ER relation that the file is based upon. To illustrate, suppose I have a visual-demonstrative file, based on my current visual relation with a bird, collecting information gathered through that relation. That visual-demonstrative file can also host information that is not acquired through my visual relation to the bird, as when a companion tells me something about the bird (77). However, the reference of the file's contents is determined by the context and the ER relation the file is based on, so the reference of the file is the unique source of the visual information in the file.

File types are characterized by their functional role in thought (246). Recanati specifies aspects of these functional roles in considerable detail. For example, Recanati considers what happens to a thinker's files when she comes to form an identity judgement (that is, she judges that $a=b$ ). Suppose a thinker starts with a file $\mathrm{F}_{\mathrm{a}}$ about $a$, and a file $\mathrm{F}_{\mathrm{b}}$ about $b$. If she judges that $a=b$, then (depending on what kind of ER relations $F_{a}$ and $F_{b}$ are based on) she will either merge $F_{a}$ and $\mathrm{F}_{\mathrm{b}}$ to form a single file, or she will link $\mathrm{F}_{\mathrm{a}}$ and $\mathrm{F}_{\mathrm{b}}$. When the thinker links files, she retains two distinct files but information can flow between those files (43-53).

However, when Recanati goes into this kind of detail, it is not always clear that he has provided a clear argument for the claims he makes, nor that he has fully explored either the consequences of his position or alternative ways of developing the mental files account of singular thought. And Recanati appears to hold back altogether from finalizing some of the most important details of any account of mental files. These tendencies can be illustrated by considering what Recanati says about an important component of an account of mental files: the identity conditions on mental files. 


\section{The importance of identity conditions}

File identity plays an important role in mental file accounts of singular thought. We are interested in mental files because they are supposed to play the 'mode of presentation' role. Part of the mode of presentation role is to account for cognitive significance phenomena (viii). For example, we are supposed to be able to explain how John rationally believes both that Hesperus is a star and Phosphorus is a planet by saying that John has two distinct files on the planet Venus. The Hesperus-file and Phosphorus-file are both internally consistent (so John is rational), but they are mutually inconsistent. This kind of explanation turns on the distinction between a thinker having two mental files on a single object, and one mental file on a single object.

And file identity is particularly important for Recanati because he emphasises distinctions such as that between linking and merging files. To distinguish linking from merging just is to distinguish cases where an identity judgement results in a single file from cases where an identity judgement results in two files with information moving between the two.

However, as I argue in Section 2, Recanati does not offer a satisfactory account of the identity conditions on files. We might think that this isn't such a problem - after all, we regularly manage to distinguish cases where there is one person in the room from cases where there are two persons in the room without the benefit of an adequate account of the identity conditions on persons. But the cases of persons and files aren't analogous: we have an independent grasp on persons beyond the philosophical theory of persons. But we have no better grasp on mental files than that provided by the philosophical theory of files. The proponent of mental files cannot rely on any independent understanding of what it is to have one file or two distinct files, but must supply this understanding as part of the theory.

To make questions about the identity conditions on mental files clearer, use the idea of 'co-filing'.

Pieces of information $i$ and $j$ are co-filed if and only if there is some file such that $i$ and $j$ are both members of that file. 
My question is: under what conditions are pieces of information $i$ and $j$ co-filed at time $t ?^{3}$ As Recanati points out (96), we can ask about co-filing at two levels. At the level of symptoms, we ask: what are the symptoms of $i$ and $j$ being co-filed? At the level of explanation, we ask: what does explain those symptoms? That is, what does explain the fact that $i$ and $j$ are co-filed?

Files are supposed to be psychologically-realised clusters of information, so it seems likely that significant progress in the question of explanation will be made only with the help of psychology. Therefore, my focus will be on the symptoms of co-filing.

\section{Identity conditions in Recanati's Mental Files}

Recanati offers an initial account of the identity conditions on files:

'To say that there are two distinct mental files is to say that information in one file is insulated from information in the other file. Files are a matter of information clustering. Clustering takes place when all the information derives from the same source, through the same ER relation...' (42)

But this answer is only provisional. It is merely a norm that information in a file derives from a single source (that is, that co-filed information originates in a single object). In practice, co-filed information may derive from multiple objects or from no object at all (63). And linking files overcomes the informational-insulation of the linked files even whilst they retain their distinct identities (43).

Although Recanati's initial account of the identity conditions on files is only provisional, he does not go on to give a clear indication of what his final account is. And as I argue in Sections 3 and 4, if we explore plausible routes Recanati could take, we find no account of identity conditions compatible with all of Recanati's other claims about mental files.

To structure the plausible routes available to Recanati for a final account of cofiling, consider how we interpret his claim that: ${ }^{4}$

\footnotetext{
${ }^{3}$ From now on, I'll take the qualification 'at time $t$ ' as read.

${ }^{4}$ This quotation is from a section outlining a now-abandoned response to circularity objections to file accounts. However, Recanati indicates that the response was abandoned because it gives only a symptom of co-filing without ex-
} 
'[T]wo pieces of information occur in the same file just in case the subject uses them (or is disposed to use them) in a certain way, namely, in an 'integrated' manner. [Footnote - For example, the subject who has the predicates 'is well-read' and 'is bald' in his Cicero-file is thereby disposed to infer that some bald man is well-read.]' (96)

I assume that Recanati's examples indicate that treating information in an integrated manner means being disposed to reason as if that information is about the same thing. ${ }^{5}$ So two pieces of information occur in the same file just in case the subject is disposed to reason as if that information is about the same thing.

However, we should remember that Recanati distinguishes between a weak and strong form of reasoning as if the information is about the same thing. It is not clear which one he takes to be a symptom of cofiling.

To understand the weak/strong distinction, we need to understand Recanati's distinction between (i) judgments of identity and (ii) presumptions of identity. When a thinker comes to judge that $a=b$, that thinker will be disposed to reason as if information about $a$ is about the same thing as information about $b$, via the identity premise $a=b$. For example, if a thinker has the information Cicero is well-read and Tully is bald, and judges that Cicero=Tully, then she is disposed to infer that some bald man is well-read, but only in virtue of the identity judgement Cicero=Tully.

But Recanati rehearses Cambell's 1998 argument that as well as judgments of identity, there must also be presumptions of identity. Presumptions of identity allow a thinker to 'trade on identity', that is to treat information as if it is about the same thing without any explicit or implicit identity judgement. To illustrate: a thinker is using a presumption of identity when she has the information Cicero is well-read and Cicero is bald, and is disposed to infer that some bald man is well-read without employing any identity judgement at all.

On the weak form of what it is for information to be used in an 'integrated manner', information $i$ and $j$ is used in an integrated

plaining it — not because it got the symptoms of co-filing wrong.

${ }^{5}$ At least for information that is at most about one thing. Matters become more complex when we consider reasoning that is about more than one thing. Following Recanati (50), I abstract away from such concerns. 
manner when the thinker is disposed to reason as if $i$ and $j$ are about the same thing, regardless of whether this is a result of a judgement of identity or a presumption of identity. On the strong form, $i$ and $j$ is used in an 'integrated manner' only when the thinker is disposed to reason as if $i$ and $j$ are about the same thing in virtue of a presumption of identity.

These distinct forms correspond to two ways we can interpret Recanati's suggestion that $i$ and $j$ occur in the same file just in case the thinker is disposed to use $i$ and $j$ in an 'integrated manner'.

The weak reading gives one possible answer to my question about the identity conditions of mental files:

CF-1 $i$ and $j$ are co-filed if and only if the thinker is disposed to reason as if $i$ and $j$ are about the same thing (whether in virtue of a presumption or judgement of identity).

But the strong reading gives a different account of identity conditions:

CF-2 $i$ and $j$ are co-filed if and only if the thinker is disposed to reason as if $i$ and $j$ are about the same thing in virtue of a presumption of identity.

But neither CF-1 nor CF-2 are obviously compatible with all of Recanati's other claims about files.

\section{Difficulties with the weak reading (CF-1)}

Given close connections between a thinker's disposition to reason as if $i$ and $j$ are about the same thing and her being said to take it to be the case that $i$ and $j$ are about the same thing, it appears that Recanati had CF-1 or something very like it in mind when he wrote:

'Two pieces of information go into the same file if they are taken to concern the same object.' (101)

However, CF-1 sits unhappily with Recanati's claim that mental files are reference-determining modes of presentation (viii). In outline, there is reason to think that if files are reference-determining modes of presentation, then all information in a single file is coreferential in a particularly strong, 'de jure' way. But CF-1 implies that not all 
information in a file is de jure coreferential.

Recanati characterizes de jure coreference between linguistic expressions: ${ }^{6}$

'I characterize de jure co-reference in terms of a priori knowledge of (conditional) co-reference: two terms are de jure co-referential just in case anyone who understands the utterance in which they occur knows that they co-refer if they refer at all.' (110)

Recanati also discusses de jure coreference in thought, and between pieces of information (e.g. 94, 120), but gives no explicit characterization of this. Extrapolating from his characterization of de jure coreference for linguistic terms gives a (very rough) characterization of de jure coreference for information:

$i$ and $j$ de jure corefer if and only if the thinker knows a priori that if $i$ and $j$ are about something they are about the same thing. ${ }^{7}$

It is not the case that: if I take $i$ and $j$ to be about the same thing or reason as if $i$ and $j$ are about the same thing, then $i$ and $j$ are de jure coreferential. Suppose I am in the garden. I hear a bird singing and also see a bird singing. I gather $p$ from the seen-bird and $q$ from the heard-bird. After leaving the garden I judge that-heard bird $=$ thatseen bird. In virtue of this identity judgement, I take it that $p$ and $q$ are about the same thing, and I am disposed to reason as such. But I retain my ability to think independently about that-heard bird and that-seen bird, that is, it remains possible that $p$ and $q$ are about different birds. ${ }^{8}$ Merely judging that that-heard bird $=$ that-heard bird does not grant me a priori knowledge that $p$ and $q$ are about the same thing (if about anything at all). Hence $p$ and $q$ are not de jure coreferential.

${ }^{6}$ For further discussion of how best to characterize de jure coreference, see Pinillos 2011 and Goodsell (forthcoming).

${ }^{7}$ Again, I abstract away from considering information which is about more than one object.

${ }^{8}$ Recanati and I agree that (at least at first) I retain my ability to think independently of that-seen bird and that-heard bird (45). My own evidence for this position is just the fact that if it turned out that the identity judgement that-seen bird $=$ that-heard bird were false, I would have a false belief of the seen-bird that it is the same as the heard-bird, and of the heard-bird that it is the same as the seen-bird. Those false beliefs are only available if I retain the ability to think independently of the heard-bird and the seen-bird. 
But by CF-1, because my identity judgement that-heard bird $=$ thatseen bird disposes me to reason as if $p$ and $q$ are about the same thing, the identity judgement results in $p$ and $q$ being stored in the same mental file. ${ }^{9}$ None of the considerations I raised to reach this conclusion turn on the irrationality of the thinker, so putting the pieces together, we find that if we adopt CF-1, we must accept that a rational thinker can have co-filed information that is not de jure coreferential.

This conclusion corresponds to Recanati's claim that if information-pieces $i$ and $j$ are in the same file as the result of information flowing between linked-files, $i$ and $j$ are not de jure coreferential, but if $i$ and $j$ occur in the same file without a prior linking operation, they are de jure coreferential (94-95).

However, I will show that there is reason to think that if we understand 'a priori knowledge' in a way that allows a priori knowledge of conditional coreference to be widespread, then so long as files are reference-determining modes of presentation, all information cofiled by a rational thinker must be de jure coreferential.

Thinkers rarely think explicitly about their information. This means that if all information which occurs in the same file without the benefit of a prior linking operation is to count as de jure coreferential, the a priori knowledge required for de jure coreference must be highly idealized, relying on the thinker's disposition to use that information rather than her explicit beliefs about the information. A very plausible option is that a thinker counts as a priori knowing that $i$ and $j$ are about the same thing (if they are about anything at all) if (i) $i$ and $j$ are about the same thing (if they are about anything at all), and (ii) the thinker acts as if (i) holds.

Recanati gives only a brief introduction to modes of presentation, largely relying on his audience's familiarity with the idea.

\footnotetext{
${ }^{9}$ By CF-1, $p$ and $q$ must be stored in the same file. On Recanati's picture, I retain two distinct files on the bird (because the bird is still given in two distinct ways, one in auditory memory and one in visual memory). To reconcile these claims, we must suppose $p$ and $q$ are both stored in two files. That is, the very same pieces of information are simultaneously in two files (rather than simply duplicated in each file). According to CF-1, the only way a thinker can retain two separate files of duplicate information is if she does not reason as if that duplicate information is about the same thing.
} 
'Modes of presentation are supposed to account for 'cognitive significance', for clustering/coordination of information, and for reference determination.' (viii)

The reference-determining role of modes of presentation means that when $i$ and $j$ are associated with the same mode of presentation, $i$ and $j$ are about the same thing (if about anything at all).

'Cognitive significance' considerations relate to how a rational thinker might have apparently inconsistent attitudes (for example, when a thinker sincerely assents to 'Hesperus is bright' whilst doubting that 'Phosphorus is bright'). The suggestion is that it is rational to take apparently inconsistent attitudes just so long as those attitudes are associated with different modes of presentation. The idea is that a thinker is rationally licensed to treat those attitudes as potentially about different objects just in case they are associated with different modes of presentation. But then the thinker is only rationally licensed to treat $i$ and $j$ as potentially being about different objects if they are associated with different modes of presentation. ${ }^{10}$ So if a rational thinker associates $i$ and $j$ with the same mode of presentation, she will not behave as if $i$ and $j$ are potentially about different objects, but she will behave as if it is the case that $i$ and $j$ are about the same thing (if about anything at all).

Putting the pieces of the mode of presentation role together, we find that if a rational thinker associates $i$ and $j$ with the same mode of presentation, then (i) $i$ and $j$ are about the same thing (if they are about anything at all), and (ii) the thinker acts as if (i) holds. But this is enough to meet the conditions for a priori knowledge of conditional coreference. So if $i$ and $j$ are associated with the same mode of presentation, then $i$ and $j$ are de jure coreferential. And this means that if, as CF-1 implies, not all information in the same mental file is de jure coreferential, files cannot play the mode of presentation role.

CF-1 is incompatible with files playing the mode of presentation role. The fact that files fill the mode of presentation role is central to our interest in files, making this a significant difficulty with CF-1. And even rejecting CF-1, there is still a difficulty for Recanati: he

\footnotetext{
${ }^{10}$ A corollary of this position is that if $i$ and $j$ start out in the same mental file, to suppose that $i$ and $j$ are about different things, the thinker must move $i$ and $j$ to distinct files.
} 
explicitly claims that it is not the case that if $i$ and $j$ are in the same mental file, then $i$ and $j$ are de jure coreferential (94-95). And it is this claim that seems inconsistent with files playing the mode of presentation role.

This line of argument could be resisted by providing a suitable alternative account of the a priori knowledge required for de jure coreference, or of de jure coreference itself. However, although these ideas play an important role in Recanati's Mental Files, they are not fully explained, making it difficult to see what the suitable alternative account would be.

\section{Difficulties with the weak reading (CF-2)}

The weak reading of the claim that $i$ and $j$ are co-filed just in case the thinker is disposed to treat them in an 'integrated manner' gave CF-1, which turned out to be incompatible with files playing the mode of presentation role. We might hope for more success with the strong reading (CF-2).

CF-2 $i$ and $j$ are co-filed if and only if the thinker is disposed to reason as if $i$ and $j$ are about the same thing in virtue of a presumption of identity.

In places, Recanati appears to have something like CF-2 in mind. Introducing the distinction between presumptions and judgments of identity, Recanati writes:

'this gives us a criterion for telling apart the cases in which there is a single file and the cases in which there are two. If the subject 'trades upon identity' and proceeds to integrate various pieces of information directly, without appealing to a further identity premise, that means that there is a single mode of presentation.' (83)

How we evaluate CF-2 depends on how we understand 'presumption of identity'. One option (the information-gathering option) is that there is a presumption of identity between $i$ and $j$ just in case the thinker is disposed to reason as if $i$ and $j$ are about the same object without ever having formed an identity judgement implying that $i$ and $j$ are about the same object, and has never called into question whether $i$ and $j$ are about the same object. The other option (the current-reasoning option) is that there is a presumption of identity between $i$ and $j$ just 
if the thinker is disposed to reason as if $i$ and $j$ are about the same object without using an additional premise implying that $i$ and $j$ are about the same thing. The disambiguations come apart in at least one direction if it is possible that though $i$ and $j$ were initially treated as about the same thing in virtue of an identity judgement, that identity judgement can become so embedded in the thinker's reasoning that she is disposed to reason as if $i$ and $j$ are about the same thing without deploying an additional identity premise in her reasoning.

Recanati's own focus on whether a thinker has ever made an identity judgement suggests the information-gathering understanding. But Campbell's 1988 argument, reiterated by Recanati, treats presumptions of identity as a kind of reasoning, suggesting the currentreasoning understanding.

The disambiguations of 'presumption of identity' give corresponding disambiguations of CF-2:

$\mathrm{CF}-2_{\mathrm{ig}} i$ and $j$ are co-filed if and only if the thinker is disposed to reason as if $i$ and $j$ are about the same thing in virtue of an information-gathering presumption of identity between $i$ and $j$.

$\mathrm{CF}-2_{\text {cr }} i$ and $j$ are co-filed if and only if the thinker is disposed to reason as if $i$ and $j$ are about the same thing in virtue of a currentreasoning presumption of identity between $i$ and $j$.

CF-2 ${ }_{\text {ig }}$ runs into trouble with file merging and linking. Judgements of identity result in files being linked or merged. Merging results in originally non-co-filed information being co-filed (46). And linking is supposed to enable information to flow from one file into another.

'Now, when two distinct files are linked... information from one file can flow freely into the other, and be integrated with the information there.' (94)

CF- $2_{\text {ig }}$ is ruled out as an option for Recanati, and for anyone who allows that files sometimes merge. Information pieces $i$ and $j$ may end up co-filed even though the thinker is only disposed to reason as if $i$ and $j$ are about the same thing because of a prior judgement of identity.

Moreover, CF-2 $2_{\text {ig }}$ is independently unattractive as an account of the identity conditions on mental files. If mental files are supposed to capture and account for how we reason now, their identity condi- 
tions should not focus on how information was gathered, but rather on what can be done now with that information.

This leaves CF-2 $2_{\text {cr }}$ CF-2 looks to be the most attractive available account of file-identity. It links identity conditions on files to how we reason now. And importantly, there appears to be no reason to think that CF- $2_{\text {cr }}$ allows for $i$ and $j$ to be co-filed without being de jure coreferential. And more generally there appears to be no argument that $\mathrm{CF}-2_{\text {cr }}$ gives identity conditions on files that are incompatible with files playing the mode of presentation role.

However, although it appears to have been possible to reconstruct an acceptable account of the identity conditions on files, it is not clear that CF-2 is consistent with all of Recanati's other claims.

First, CF-2 cr appears to conflict with Recanati's claim, quoted above, that merely taking $i$ and $j$ to be about the same thing is sufficient for their being co-filed (101). Second, CF-2 is incompatible with his suggestion that when $i$ and $j$ are co-filed due to linking, there is no presumption of identity between $i$ and $j$ (94-95). According to $\mathrm{CF}-2_{\text {cr }}$, co-filing requires presumptions of identity. And third, CF-2 2 appears to conflict with Recanati's claim that when 'two distinct files are linked, information is allowed to flow freely between them' (94). Instead, CF-2 suggests that linking could only result in information moving from one file to another when the identity judgement is so embedded that the thinker starts using current-reasoning presumptions of identity in her reasoning.

However, it is not clear how problematic this is for Recanati's account of files. Recanati does not provide clear arguments for the three claims about mental representation that $\mathrm{CF}_{-} 2_{\text {cr }}$ is incompatible with, and as discussed, he does not explore fully all their consequences. ${ }^{11}$ As a result, it is not clear to what extent these claims

\footnotetext{
${ }^{11}$ The closest that there is to an explanation is that to count as concepts, files must satisfy the Generality Constraint (see Evans 1982), that is 'a file should be hospitable to any predicative concept in the subject's possession' (65). We might attempt to meet this constraint by saying that a file can host information acquired through linking as well as through the ER relation the file is based on. But arguably, this does not help the file to meet the generality constraint - if a file can only host information sourced through linking or the ER relation, we still have some limitations on what predicates the file can contain. For example, if I never link my 'moon' file to a file containing '- is a mammal', I will never be able to host the
} 
are important to Recanati's picture, and to what extent they can be abandoned without cost.

\section{Conclusion}

I have argued that it is important for Recanati to have an account of the identity conditions on files, and have shown that Recanati does not have a clear final account of these identity conditions. I have also shown that neither of the plausible routes Recanati could take are fully compatible with his other claims about mental files, though I have pointed out that the fact that Recanati has not argued for all details of his mental file theory means that it is not clear whether this incompatibility is a significant concern.

As Recanati rightly points out:

'We investigate the phenomena by constructing models for them, and we follow the model where it leads to see, precisely, where it leads.' (50)

But this does not mean that we should construct just any model. We need some argument for why we construct the model as we do, or at least a comparison of the model with its competitors. Helpfully, Recanati finishes the book with a comparison of his semantic framework with alternative frameworks purporting to explain the same semantic phenomena without mental files. But behind Recanati's semantic framework is what appears to be a substantive and in places detailed theory about mental representation. It would have been helpful for Recanati to include more discussion of the relative strengths of this part of the mental file picture — or a disclaimer that despite appearances his is not a substantive theory of mental representation, and with it an explanation of what can be retained of the semantic framework without taking 'mental files' to be a substantive psychological posit. ${ }^{12}$

predicate '- is a mammal' in my 'moon' file. The Generality Constraint is better met just by allowing that a file can host any predicate in virtue of the thinker supposing that the subject of the file satisfies the predicate.

${ }^{12}$ I am grateful to John Hawthorne, Daniel Morgan, David Papineau and James Studd for their helpful comments and discussion of earlier drafts. 
Thea Goodsell

Magdalen College

Oxford, OX1 4AU

United Kingdom

thea.goodsell@magd.oxon.org

\section{References}

Campbell, John. 1988. Is Sense Transparent? Proceedings of the Aristotelian Society, 88: 273-292.

Dickie, Imogen. 2010. We are Acquainted with Ordinary Things. In New Essays on Singular Thought, ed. by Robin Jeshion, 213-245. Oxford: Oxford University Press.

Evans, Gareth. 1982. The Varieties of Reference. Oxford: Clarendon Press.

Forbes, Graeme. 1990. The Indispensability of Sinn. The Philosophical Review, 99(4): 535-563.

Goodsell, Thea. Forthcoming. Is De Jure Coreference Non-Transitive? Philosophical Studies.

Heim, Irene. 1983. File Change Semantics and the Familiarity Theory of Definiteness. In Meaning, Use, and Interpretation of Language, ed. by Rainer Bäuerle, Christoph Schwarz and Arnim von Stechow, 164-190. Walter de Gruyter.

Heim, Irene. 1988. The Semantics of Definite and Indefinite Noun Phrases. Outstanding Dissertations in Linguistics. New York, NY: Garland Publishing.

Jeshion, Robin. 2010. Singular Thought: Acquaintance, Semantic Instrumentalism, and Cognitivism. In New Essays on Singular Thought, ed. by Robin Jeshion, 105-140. Oxford: Oxford University Press.

Kahneman, Daniel and Treisman, Anne. 1984. Changing Views of Attention and Automaticity. In Varieties of Attention, ed. by Raja Parasuraman and David R. Davies, 29-61. Orlando: Academic Press.

Kahneman, Daniel, Treisman, Anne and Gibbs, Brian J. 1992. The Reviewing of Object Files: Object-Specific Integration of Information. Cognitive Psychology, 24(2): 175-219.

Perry, John. 1980. A Problem About Continued Belief. Pacific Philosophical Quarterly, 61(4): 317-332.

Pinillos, Ángel. 2011. Coreference and Meaning. Philosophical Studies, 154(2):301324.

Segal, Gabriel. 2001. Two Theories of Names. Mind \& Language, 16(5):547-563.

Strawson, Peter F. 1974. Subject and Predicate in Logic and Grammar. London: Methuen \& Co. 